\title{
Mild and efficient catalytic method for $\alpha$-trimethylsilyl ketones
}

\author{
Chunrui Sun, Jingwei Li, Silviya Demerzhan, and Daesung Lee* \\ Department of Chemistry, University of Illinois at Chicago, 845 West Taylor Street, Chicago, \\ Illinois 60607-7061 USA \\ E-mail: dsunglee@uic.edu
}

\section{Dedication to Prof. Siegfried Blechert on the occasion of his $65^{\text {th }}$ birthday}

\begin{abstract}
A mild, efficient and convenient method for the synthesis of $\alpha$-silyl ketones from corresponding aldehydes and trimethylsilyldiazomethane in the presence of a catalytic amount of indium(III) chloride has been developed.
\end{abstract}

Keywords: Indium chloride, $\alpha$-silyl ketones, trimethylsilyldiazomethane, Lewis-acids, insertion, homologation

\section{Introduction}

$\alpha$-Silyl ketones are useful building blocks in organic synthesis because they are substrates for many regio- and stereoselective reactions to construct carbon-carbon and carbon-heteroatom bonds in high stereoselectivities. ${ }^{1}$ Generally, $\alpha$-silyl ketones can be synthesized through several methods including condensation of esters or acyl halides with $\alpha$-silylated organolithium ${ }^{2}$ or organomagenesium $^{3}$ reagents, reaction of $\alpha$-silyl esters with Grignard reagents, ${ }^{4}$ oxidation of vinylsilanes followed by rearrangement, ${ }^{5}$ and deprotonation-silylation of corresponding hydrazones, ${ }^{6}$ and $\mathrm{O} \rightarrow \mathrm{C}$ silyl migration strategy starting from $\alpha$-bromoketones. ${ }^{7}$ Some of these methods are versatile yet milder and more efficient methods are highly needed.

We envision that an appropriate Lewis-acid may be used as catalyst in the reaction of trimethylsilyldiazomethane and aldehydes to form $\alpha$-silyl ketones. In this method, a Lewis acidactivated aldehyde $\mathbf{A}$ would undergo a nucleophilic addition by trimethylsilyldiazomethyl carbon in $\mathbf{B}$ to afford diazonium intermediate $\mathbf{C}$. This intermediate then will undergo a 1,2-hydride shift with a concomitant extrusion of nitrogen to form the desired $\alpha$-silyl ketone product and the regenerated catalyst (Scheme 1). Many homologation reactions of cyclic ketones with diazomethane and its derivatives, all of which share the same mechanistic paradigm, have been reported in the literature ${ }^{8}$ but the homologation of aldehyde with trimethylsilyldiazomethane to selectively generate $\alpha$-silyl ketones is rare. ${ }^{9}$ Probably, this is because not only the $\alpha$-silyl ketones 
have a strong propensity to rearrange into the corresponding silyl enol ether but also the zwitterionic intermediate $\mathbf{D}$ tends to form a carbene intermediate $\mathbf{E}$ due to the formation of a strong silicon-oxygen bond, thereby generating silyl enol ether. Furthermore, relatively unstable $\alpha$-silyl ketone products may further undergo secondary transformations to generate the corresponding protodesilylated methyl ketones, which must be suppressed to maximize the yield of $\alpha$-silyl ketones. In this article, we report an indium(III) chloride-catalyzed reaction between aldehydes and trimethylsilyldiazomethane as a useful method for the selective synthesis of $\alpha$-silyl ketones where indium(III) chloride was found to be a uniquely effective catalyst compared to many other Lewis acids.

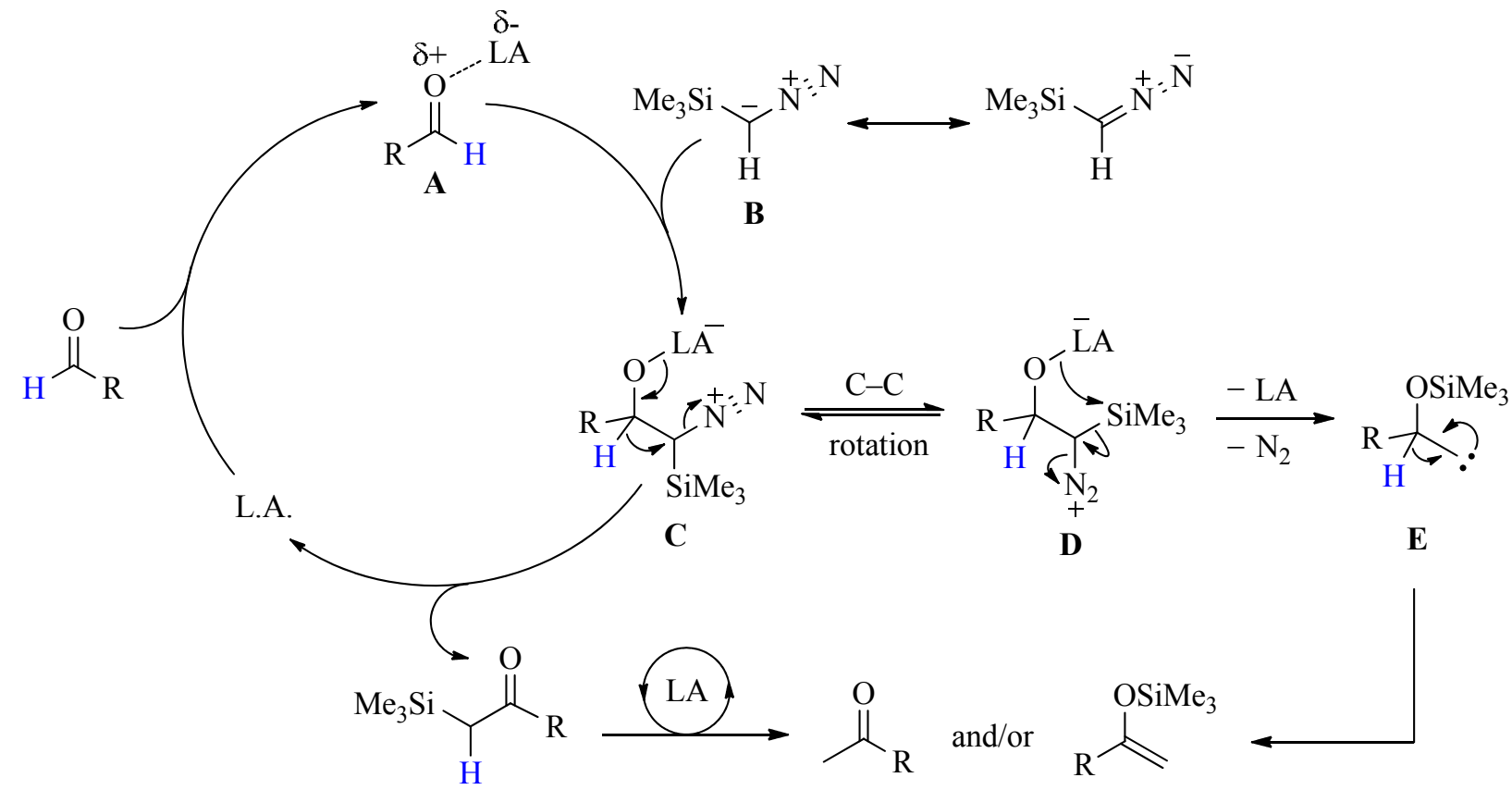

Scheme 1. Proposed mechanism for Lewis acid-catalyzed synthesis of $\alpha$-trimethylsilyl ketones.

\section{Results and Discussion}

First, we selected 4-cis-decenal $\mathbf{1}$ as a model substrate to examine the reaction scope and the performance of different Lewis acids (Table 1). These reactions were carried out at room temperature using $2 \mathrm{~mol} \%$ Lewis acid in methylene chloride. All Lewis acids tested afforded either $\alpha$-silyl ketone 2 and/or silyl enol ether 3 in 30 minutes. A boron-based Lewis acid such as $\mathrm{BF}_{3} \cdot \mathrm{Et}_{2} \mathrm{O}$ exhibited a high reactivity to generate $\mathbf{2}$ and small amount of methyl ketone $\mathbf{4}$, which, we believe, resulted from $\alpha$-silyl ketone 2 via a protodesilylation under the reaction conditions (entry 1). Metal triflates such as $\mathrm{Hf}(\mathrm{OTf})_{3}, \mathrm{In}(\mathrm{OTf})_{3}$ and $\mathrm{Sc}(\mathrm{OTf})_{3}$ afforded silyl enol ether 3 as the major product, which is assumed to be a secondary product from $\alpha$-silylketone 2 via $\mathrm{C} \rightarrow \mathrm{O}$ 
silyl migration catalyzed by the Lewis acids. It is worthwhile to note that $\mathrm{Sc}(\mathrm{OTf})_{3}$-catalyzed reaction generated silyl enol ether exclusively in quantitative yield (entry 8). ${ }^{8 c, 10}$ Other triflates such as $\mathrm{Mg}(\mathrm{OTf})_{2}$ and $\mathrm{Zn}(\mathrm{OTf})_{2}$ did not promote the formation of the silyl enol ether, yet low yield of 2 and formation of $\mathbf{4}$ was observed with these catalysts (entries 7 and 12). Halide-based Lewis acids, in general, showed better catalytic performance than the corresponding triflates, and among the Lewis acids examined, indium(III) chloride $\left(\mathrm{InCl}_{3}\right)$ was found to be the best catalyst, leading to complete conversion and high selectivity for the desired $\alpha$-silyl ketone 2 , minimizing its rearrangement to silyl enol ether $\mathbf{3}$ or protodesilylation to methyl ketone 4.

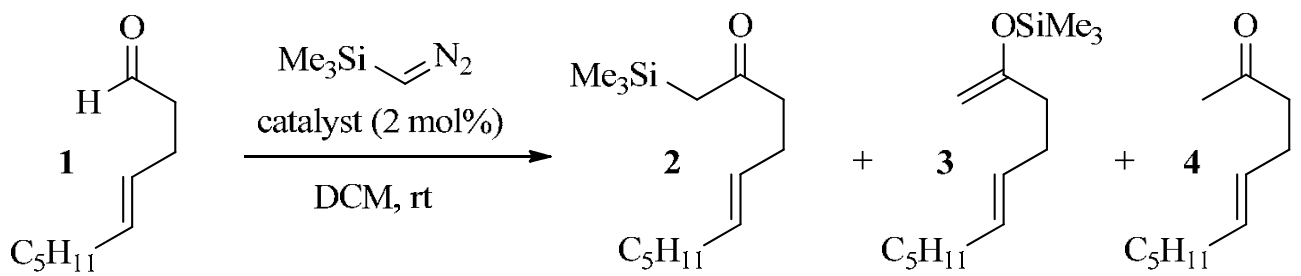

Table 1. Optimization of Reaction Conditions ${ }^{\mathrm{a}}$

\begin{tabular}{|c|c|c|c|c|c|}
\hline \multirow{2}{*}{ Entry } & \multirow{2}{*}{ Catalyst } & \multirow{2}{*}{$\begin{array}{c}\text { Conversion } \\
(\%)\end{array}$} & \multicolumn{3}{|c|}{ Yields $^{\mathrm{b}}(\%)$} \\
\hline & & & 2 & 3 & 4 \\
\hline 1 & $\mathrm{BF}_{3} \cdot \mathrm{Et}_{2} \mathrm{O}$ & 100 & 97 & 0 & 3 \\
\hline 2 & $\mathrm{Hf}(\mathrm{OTf})_{3}$ & 100 & 4.5 & 72 & 16 \\
\hline 3 & $\operatorname{In}(\mathrm{OTf})_{3}$ & 100 & 0 & 83 & 15 \\
\hline 4 & $\mathrm{InCl}_{3}$ & 100 & 98 & $\mathbf{0}$ & $\mathbf{0}$ \\
\hline 5 & $\mathrm{InBr}_{3}$ & 100 & 73 & 11 & 16 \\
\hline 6 & $\mathrm{MgBr}_{2}$ & 88 & 88 & 0 & 0 \\
\hline 7 & $\operatorname{Mg}(\mathrm{OTf})_{2}$ & 6 & 3 & 0 & 3 \\
\hline 8 & $\mathrm{Sc}(\mathrm{OTf})_{3}$ & 100 & 0 & 98 & 0 \\
\hline 9 & $\operatorname{Sm}(\mathrm{OTf})_{3}$ & 100 & 71 & 3 & 23 \\
\hline 10 & $\mathrm{SnCl}_{2}$ & 2 & 2 & 0 & 0 \\
\hline 11 & $\mathrm{ZnCl}_{2}$ & 100 & 84 & $<1$ & 15 \\
\hline 12 & $\mathrm{Zn}(\mathrm{OTf})_{2}$ & 27 & 12 & 0 & 9 \\
\hline
\end{tabular}

${ }^{a}$ Aldehyde $(0.5 \mathrm{mmol})$, trimethylsilyldiazomethane $(0.55 \mathrm{mmol})$ and catalyst $(2 \mathrm{~mol} \%)$ in DCM (1 mL). ${ }^{\text {}} \mathrm{NMR}$ yields based on internal standard 1,2,4,5-tetrabromobenzene.

Next, we turned our attention to expanding the substrate scope using the optimized conditions (Table 2). The results show that this indium chloride-catalyzed reaction tolerates diverse functional groups including alkene (entries 1,2 and 4), alkyne (entry 3), ether (entries 911 ), acetal (entry 12), and ketone (entries 13). In case of the reaction with benzaldehydes, 
however, $\alpha$-silyl ketone products were obtained in slightly lower yields due to the formation of an epoxide byproduct (entries 6-8). Although Fournier and coworkers recently reported that the reaction between ethyl diazoacetate and 2-aminobenzaldehydes in the presence of $\mathrm{BF}_{3} \cdot \mathrm{OEt}_{2}$ afforded indole through [1,2]-aryl shift, ${ }^{11}$ no [1,2]-aryl shift was observed under current conditions with these aldehydes. The alkoxy group on the $\alpha$-position of the aldehyde did not cause deleterious effect for the methylene insertion, thus $\alpha$-silyl ketone $\mathbf{6 j}$ and $\mathbf{6 k}$ were obtained in excellent yields (entries 10 and 11). The substrate with both aldehyde and ketone moiety gives $\alpha$-silyl ketone $6 \mathrm{~m}$ without the participation of the ketone moiety (entry 13). An $\alpha$-branched aldehyde 2-methylpentanal also efficiently afforded the corresponding $\alpha$-silyl ketone $\mathbf{6 n}$ without incident (entry 14).

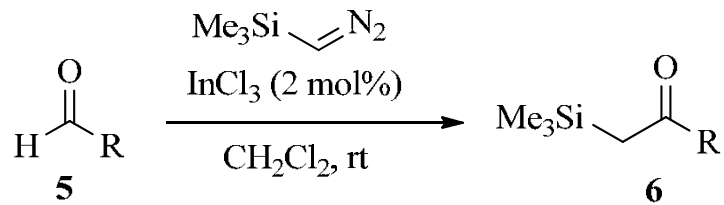

Table 2. Indium(III)-catalyzed synthesis of $\alpha$-trimethylsilyl ketones

Entry


Table 2. Continued

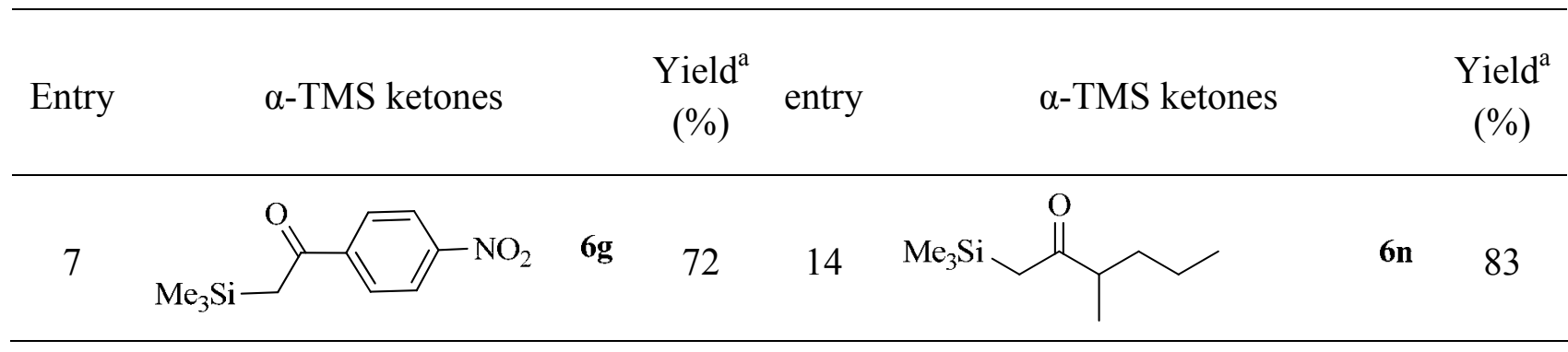

${ }^{a}$ NMR yields based on internal standard 1,2,4,5-tetrabromobenzene.

The synthetic utility of this method was further demonstrated by one-pot synthesis of $\alpha$-silyl ketones followed by their conversion to silylated cyclopropene $\mathbf{7}$ or to a cyclic allylsilane $\mathbf{8}$ (Scheme 2). ${ }^{12}$

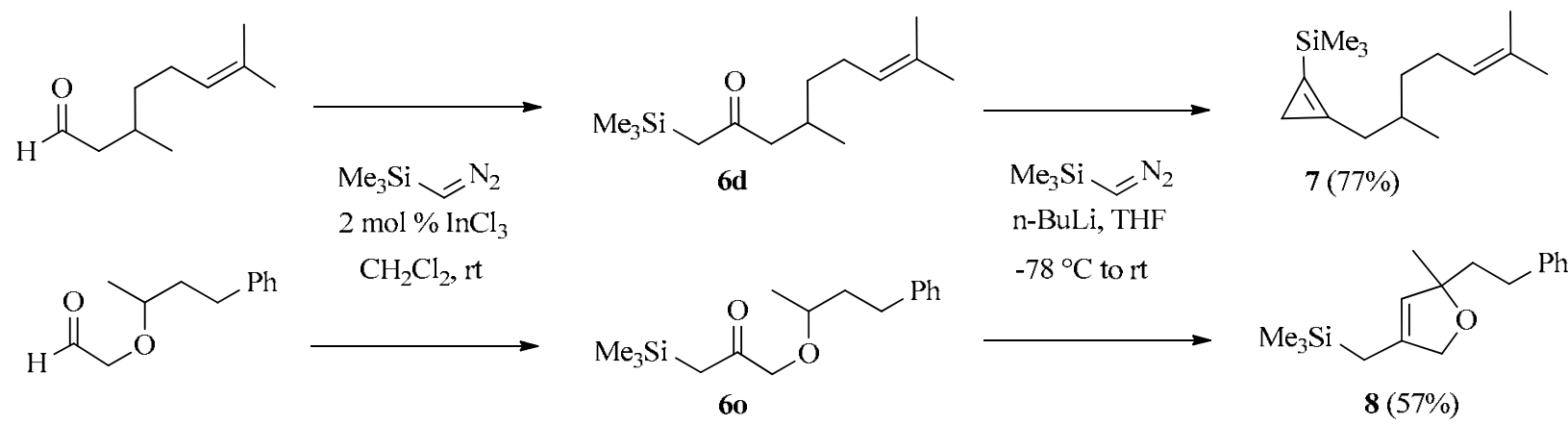

Scheme 2. Formation of $\alpha$-silyl ketones and their $\mathrm{C}-\mathrm{Si}$ and $\mathrm{C}-\mathrm{H}$ insertion reactions.

\section{Conclusions}

In summary, we have developed an indium(III) chloride-catalyzed reaction between aldehydes and trimethylsilyldiazomethane as a new and effective method for the synthesis of $\alpha$-silyl ketones. Indium(III) chloride was found to be uniquely effective and selective to provide high yield of the desired $\alpha$-silyl ketones by minimizing protodesilylation and/or $\mathrm{C} \rightarrow \mathrm{O}$ silyl migration compared to other Lewis acids.

\section{Experimental Section}


General. All reactions were carried out under air, unless otherwise indicated. Compounds were purchased from Aldrich unless otherwise noted. $\mathrm{CH}_{2} \mathrm{Cl}_{2}$, THF, $\mathrm{Et}_{2} \mathrm{O}$ were purified based on standard procedures. Analytical thin layer chromatography (TLC) was performed on $0.25 \mathrm{~mm}$ E. Merck precoated silica gel 60 (particle size $0.040-0.063 \mathrm{~mm}$ ). ${ }^{1} \mathrm{H}$ NMR and ${ }^{13} \mathrm{C}$ NMR spectra were recorded on a Bruker DRX-500 spectrometer. ${ }^{1} \mathrm{H}$ and ${ }^{13} \mathrm{C}$ chemical shifts were referenced to internal solvent resonances and reported relative to $\mathrm{SiMe}_{4}$; multiplicities are indicated by $\mathrm{s}$ (singlet), d (doublet), t (triplet), q (quartet), qn (quintet), m (multiplet) and br (broad). Coupling constants, $J$, are reported in $\mathrm{Hz}$ (Hertz). Electrospray ionization (ESI) mass spectra were recorded on a Micromass LCT equipped with a time-of-flight analyzer on a Waters Micromass Q-Tof Ultima in the University of Illinois at Urbana-Champaign. Electron impact (EI) mass spectra were obtained using a Micromass AutoSpecTM.

\section{Typical procedure for the preparation of $\alpha$-trimethylsilyl ketones}

To a stirred solution of aldehyde $(0.5 \mathrm{mmol})$ and trimethylsilyldiazomethane $(0.55 \mathrm{mmol}, 2.0 \mathrm{M}$ in Ether, $0.28 \mathrm{~mL})$ in anhydrous methylene chloride $(1 \mathrm{~mL})$ at room temperature was added $\mathrm{InCl}_{3}(0.01 \mathrm{mmol}, 2.2 \mathrm{mg})$ in three portions over 10 minutes. Vigorous gas evolution was observed right away. Meanwhile TLC indicated the fully consumption of the starting material. Solvent was removed under reduced pressure to give the crude product. The yields were calculated on the basis of crude ${ }^{1} \mathrm{H}$ NMR using 1,2,4,5-tetrabromobenzene as an internal standard since $\alpha$-trimethylsilyl ketones tend to undergo protodesilylation and/or СПО silyl migration during purification on silica gel.

2. ${ }^{1} \mathrm{H}$ NMR $\left(500 \mathrm{MHz}, \mathrm{CDCl}_{3}\right) \delta 5.39-5.32(\mathrm{~m}, 1 \mathrm{H}), 5.32-5.26(\mathrm{~m}, 1 \mathrm{H}), 2.37(\mathrm{~d}, J=6.7 \mathrm{~Hz}$, $2 \mathrm{H}), 2.26(\mathrm{q}, J=7.3 \mathrm{~Hz}, 2 \mathrm{H}), 2.22(\mathrm{~s}, 2 \mathrm{H}), 2.01$ (q, $J=7.1 \mathrm{~Hz}, 2 \mathrm{H}), 1.34-1.21(\mathrm{~m}, 6 \mathrm{H}), 0.87-$ $0.85(\mathrm{~m}, 3 \mathrm{H}), 0.10(\mathrm{~s}, 9 \mathrm{H}) ;{ }^{13} \mathrm{C} \mathrm{NMR}\left(125 \mathrm{MHz}, \mathrm{CDCl}_{3}\right) \delta 208.90,131.09,127.96,44.30,38.29$, $31.52,29.35,27.14,22.58,21.83,14.08,-1.04$.

3. ${ }^{1} \mathrm{H}$ NMR $\left(500 \mathrm{MHz}, \mathrm{CDCl}_{3}\right) \delta 5.44-5.39(\mathrm{~m}, 2 \mathrm{H}), 4.04(\mathrm{dd}, J=1.7,0.6 \mathrm{~Hz}, 2 \mathrm{H}), 2.15-2.13$ (m, 2H), 2.08-2.04 (m, 2H), 1.99-1.95 (m, 2H), 1.37-1.23 (m, 6H), 0.88 (t, J=7.0 Hz, 3H), 0.20 $(\mathrm{s}, 9 \mathrm{H}) ;{ }^{13} \mathrm{C}$ NMR $\left(125 \mathrm{MHz}, \mathrm{CDCl}_{3}\right) \delta 159.00,137.73,130.85,129.28,89.93,36.70,32.57$, $31.41,30.03,29.30,22.58,14.07,0.13$.

6a. ${ }^{1} \mathrm{H}$ NMR $\left(500 \mathrm{MHz}, \mathrm{CDCl}_{3}\right) \delta 5.08-5.05(\mathrm{~m}, 1 \mathrm{H}), 2.36(\mathrm{dd}, J=9.1,5.9 \mathrm{~Hz}, 2 \mathrm{H}), 2.23(\mathrm{t}, J=$ $7.5 \mathrm{~Hz}, 2 \mathrm{H}), 2.21(\mathrm{~s}, 2 \mathrm{H}), 1.67(\mathrm{~s}, 3 \mathrm{H}), 1.62(\mathrm{~s}, 3 \mathrm{H}), 0.11(\mathrm{~s}, 9 \mathrm{H}) ;{ }^{13} \mathrm{C} \mathrm{NMR}\left(125 \mathrm{MHz}, \mathrm{CDCl}_{3}\right) \delta$ 209.31, 163.95, 132.51, 123.08, 44.38, 38.34, 25.69, 22.68, 17.65, -1.04; HRMS (ESI) calc. for $\mathrm{C}_{11} \mathrm{H}_{23} \mathrm{OSi}[\mathrm{M}+\mathrm{H}]^{+} 199.1518$, found 199.1512 .

6b. ${ }^{1} \mathrm{H}$ NMR $\left(500 \mathrm{MHz}, \mathrm{CDCl}_{3}\right) \delta 5.78(\mathrm{ddt}, J=17.0,10.3,6.7 \mathrm{~Hz}, 1 \mathrm{H}), 5.01-4.92(\mathrm{~m}, 2 \mathrm{H})$, $2.34(\mathrm{t}, J=7.4 \mathrm{~Hz}, 2 \mathrm{H}), 2.20$ (s, 2H), 2.07-2.02 (m, 2H), 1.59-1.53 (m, 2H), 1.37 (m, 2H), 0.11 (s, 9H); ${ }^{13} \mathrm{C} \mathrm{NMR}\left(125 \mathrm{MHz}, \mathrm{CDCl}_{3}\right)$ 209.53, 138.64, 114.52, 44.14, 38.18, 33.52, 28.71, 23.30, -0.95 ; HRMS (ESI) calç. for $\mathrm{C}_{11} \mathrm{H}_{23} \mathrm{OSi}[\mathrm{M}+\mathrm{H}]^{+} 199.1518$, found 199.1508 .

6c. ${ }^{1} \mathrm{H}$ NMR $\left(500 \mathrm{MHz}, \mathrm{CDCl}_{3}\right) \delta 2.45(\mathrm{~s}, 2 \mathrm{H}), 1.03-0.98(\mathrm{q}, J=8.0 \mathrm{~Hz}, 9 \mathrm{H}), 0.69-0.64(\mathrm{t}, J=$ $8.0 \mathrm{~Hz}, 6 \mathrm{H}), 0.18-0.17(\mathrm{~s}, 9 \mathrm{H}) ;{ }^{13} \mathrm{C} \mathrm{NMR}\left(125 \mathrm{MHz}, \mathrm{CDCl}_{3}\right) \delta 185.69,104.82,94.07,41.94$, $7.32,3.87,-1.21$. 
6d. ${ }^{1} \mathrm{H}$ NMR $\left(500 \mathrm{MHz}, \mathrm{CDCl}_{3}\right) \delta$ 5.08-5.05 (m, 1H), 2.34-2.10 (m, 3H), 1.98-1.90 (m, 3H), $1.68-1.61(\mathrm{~s}, 3 \mathrm{H}), 1.58(\mathrm{~s}, 3 \mathrm{H}), 1.33-1.24(\mathrm{~m}, 1 \mathrm{H}), 1.19-1.12(\mathrm{~m}, 1 \mathrm{H}), 0.88(\mathrm{q}, J=6.2 \mathrm{~Hz}, 3 \mathrm{H})$, 0.09 (s, 9H); ${ }^{13} \mathrm{C}$ NMR $\left(125 \mathrm{MHz}, \mathrm{CDCl}_{3}\right) \delta$ 209.36, 131.36, 124.43, 51.90, 38.67, 37.05, 29.07, 25.71, 25.53, 19.86, 17.66, -1.03; HRMS (ESI) calc. for $\mathrm{C}_{14} \mathrm{H}_{29} \mathrm{OSi}[\mathrm{M}+\mathrm{H}]^{+}$241.1988, found 241.1978 .

6e. ${ }^{1} \mathrm{H}$ NMR $\left(500 \mathrm{MHz}, \mathrm{CDCl}_{3}\right) \delta 2.43(\mathrm{dd}, J=6.7,3.8 \mathrm{~Hz}, 2 \mathrm{H}), 2.24(\mathrm{~d}, J=6.8 \mathrm{~Hz}, 1 \mathrm{H}), 2.17$ $(\mathrm{s}, 2 \mathrm{H}), 1.82(\mathrm{~d}, J=2.2 \mathrm{~Hz}, 2 \mathrm{H}), 1.77-1.73(\mathrm{~m}, 6 \mathrm{H}), 1.67(\mathrm{~d}, J=15.0 \mathrm{~Hz}, 2 \mathrm{H}), 1.60(\mathrm{~s}, 2 \mathrm{H}), 1.50$ $(\mathrm{d}, J=12.2 \mathrm{~Hz}, 2 \mathrm{H}), 0.08(\mathrm{~s}, 9 \mathrm{H}) ;{ }^{13} \mathrm{C} \mathrm{NMR}\left(125 \mathrm{MHz}, \mathrm{CDCl}_{3}\right) \delta$ 209.33, 48.04, 39.95, 38.95, $38.54,38.23,32.06,31.84,27.88$, -1.03; HRMS (ESI) calc. for $\mathrm{C}_{16} \mathrm{H}_{29} \mathrm{OSi}[\mathrm{M}+\mathrm{H}]^{+} 265.1988$, found 265.1982 .

6f. ${ }^{1} \mathrm{H}$ NMR $\left(500 \mathrm{MHz}, \mathrm{CDCl}_{3}\right) \delta$ 7.91-7.90 (m, 2H), $7.52(\mathrm{~m}, 1 \mathrm{H}), 7.45-7.42(\mathrm{~m}, 2 \mathrm{H}), 2.76(\mathrm{~s}$, 2H), $0.08(\mathrm{~s}, 9 \mathrm{H}) ;{ }^{13} \mathrm{C}$ NMR $\left(125 \mathrm{MHz}, \mathrm{CDCl}_{3}\right) \delta 199.58,132.60,128.44,128.31,128.24,33.70$, -0.91; HRMS (ESI) calc. for $\mathrm{C}_{16} \mathrm{H}_{28} \mathrm{ONSiNa}[\mathrm{M}+\mathrm{Na}] 287.1807$, found 287.1808.

6g. ${ }^{1} \mathrm{H}$ NMR $\left(500 \mathrm{MHz}, \mathrm{CDCl}_{3}\right) \delta 8.29-8.26(\mathrm{~m}, 2 \mathrm{H}), 8.05-8.02(\mathrm{~m}, 2 \mathrm{H}), 2.80(\mathrm{~s}, 2 \mathrm{H}), 0.07$ (s, 9H); ${ }^{13} \mathrm{C}$ NMR $\left(125 \mathrm{MHz}, \mathrm{CDCl}_{3}\right) \delta 197.78,129.30,123.77,123.39,34.29,-0.97$; HRMS (EI) calc. for $\mathrm{C}_{11} \mathrm{H}_{15} \mathrm{O}_{3} \mathrm{NSi} 237.08213$, found 237.08189 .

6h. ${ }^{1} \mathrm{H}$ NMR $\left(500 \mathrm{MHz}, \mathrm{CDCl}_{3}\right) \delta 7.41-7.38$ (m, 2H), 7.27-7.25 (m, 1H), 2.78 (s, 2H), 0.04 (s, $9 \mathrm{H}) ;{ }^{13} \mathrm{C} \mathrm{NMR}\left(125 \mathrm{MHz}, \mathrm{CDCl}_{3}\right) \delta 200.42,136.85,131.98,130.48,130.22,127.19,126.78$, 38.61, -1.04; HRMS (EI) calc. for $\mathrm{C}_{11} \mathrm{H}_{14} \mathrm{OCl}_{2} \mathrm{Si} 260.01911$, found 260.01961.

6i. ${ }^{1} \mathrm{H}$ NMR $\left(500 \mathrm{MHz}, \mathrm{CDCl}_{3}\right) \delta 7.35-7.30(\mathrm{~m}, 4 \mathrm{H}), 7.28-7.24(\mathrm{~m}, 1 \mathrm{H}), 4.55(\mathrm{~d}, J=11.7 \mathrm{~Hz}$, $1 \mathrm{H}), 4.40(\mathrm{~d}, J=11.7 \mathrm{~Hz}, 1 \mathrm{H}), 3.57-3.51(\mathrm{~m}, 1 \mathrm{H}), 2.52-2.39(\mathrm{~m}, 2 \mathrm{H}), 2.18(\mathrm{~s}, 2 \mathrm{H}), 1.84-1.70$ $(\mathrm{m}, 2 \mathrm{H}), 1.19(\mathrm{~d}, J=6.1 \mathrm{~Hz}, 3 \mathrm{H}), 0.10(\mathrm{~s}, 9 \mathrm{H}) ;{ }^{13} \mathrm{C}$ NMR $\left(125 \mathrm{MHz}, \mathrm{CDCl}_{3}\right) \delta 209.36,131.36$, $124.43,51.90,38.67,37.05,29.07,25.71,25.53,19.86,17.66,-1.03$.

6j. ${ }^{1} \mathrm{H}$ NMR $\left(500 \mathrm{MHz}, \mathrm{CDCl}_{3}\right) \delta 5.10-5.07(\mathrm{~m}, 1 \mathrm{H}), 3.88(\mathrm{~s}, 2 \mathrm{H}), 3.49$ (tq, $\left.J=6.6,3.2 \mathrm{~Hz}, 2 \mathrm{H}\right)$, $2.31(\mathrm{~s}, 2 \mathrm{H}), 2.01-1.93(\mathrm{~m}, 2 \mathrm{H}), 1.69(\mathrm{~s}, 3 \mathrm{H}), 1.61(\mathrm{~s}, 3 \mathrm{H}), 1.42(\mathrm{dq}, J=13.8,6.9 \mathrm{~Hz}, 1 \mathrm{H}), 1.36-$ $1.30(\mathrm{~m}, 1 \mathrm{H}), 1.20-1.13(\mathrm{~m}, 1 \mathrm{H}), 0.89(\mathrm{dd}, J=6.6,3.0 \mathrm{~Hz}, 3 \mathrm{H}), 0.12-0.11(\mathrm{~s}, 9 \mathrm{H}) ;{ }^{13} \mathrm{C}$ NMR $\left(125 \mathrm{MHz}, \mathrm{CDCl}_{3}\right) \delta 208.30,131.30$ 124.80, 124.72, 69.94, 37.21, 36.54, 34.21, 29.50, 25.75, 25.48, 19.54, 17.67, -0.98; HRMS (ESI) calc. for $\mathrm{C}_{16} \mathrm{H}_{32} \mathrm{O}_{2} \mathrm{NaSi}[\mathrm{M}+\mathrm{Na}]$ 307.2069, found 307.2062 .

6k. ${ }^{1} \mathrm{H}$ NMR $\left(500 \mathrm{MHz}, \mathrm{CDCl}_{3}\right) \delta 7.39(\mathrm{~d}, J=7.5 \mathrm{~Hz}, 2 \mathrm{H}), 7.32(\mathrm{t}, J=7.5 \mathrm{~Hz}, 2 \mathrm{H}), 7.27-7.22$ $(\mathrm{m}, 1 \mathrm{H}), 6.64-6.61(\mathrm{~m}, 1 \mathrm{H}), 6.29(\mathrm{dt}, J=15.9,6.1 \mathrm{~Hz}, 1 \mathrm{H}), 4.21(\mathrm{~d}, J=6.0 \mathrm{~Hz}, 2 \mathrm{H}), 3.99$ (s, 2H), $2.32(\mathrm{~s}, 2 \mathrm{H}), 0.13(\mathrm{~s}, 9 \mathrm{H}) ;{ }^{13} \mathrm{C} \mathrm{NMR}\left(125 \mathrm{MHz}, \mathrm{CDCl}_{3}\right) \delta 207.51,136.44,133.33,128.62$, 127.91, 126.55, 125.14, 75.45, 71.89, 34.19, -1.07; HRMS (EI) calc. for $\mathrm{C}_{15} \mathrm{H}_{22} \mathrm{O}_{3} \mathrm{NaSi}[\mathrm{M}+\mathrm{Na}]$ 285.1287, found 285.1281 .

61. ${ }^{1} \mathrm{H}$ NMR $\left(500 \mathrm{MHz}, \mathrm{CDCl}_{3}\right) \delta 4.46-4.41(\mathrm{~m}, 1 \mathrm{H}), 4.20-4.17(\mathrm{~m}, 1 \mathrm{H}), 3.54-3.50(\mathrm{~m}, 1 \mathrm{H})$, $2.86(\mathrm{dd}, J=17.0,5.6 \mathrm{~Hz}, 1 \mathrm{H}), 2.51(\mathrm{dd}, J=16.9,7.6 \mathrm{~Hz}, 1 \mathrm{H}), 2.24(\mathrm{~d}, J=2.7 \mathrm{~Hz}, 2 \mathrm{H}), 1.39$ (s, $3 \mathrm{H}), 1.33(\mathrm{~s}, 3 \mathrm{H}), 0.12(\mathrm{~s}, 9 \mathrm{H}) ;{ }^{13} \mathrm{C} \mathrm{NMR}\left(125 \mathrm{MHz}, \mathrm{CDCl}_{3}\right) \delta 206.94,108.61,71.87,69.67$, 48.65, 38.89, 26.92, 25.45, -1.04; HRMS (ESI) calc. for $\mathrm{C}_{11} \mathrm{H}_{22} \mathrm{O}_{3} \mathrm{NaSi}[\mathrm{M}+\mathrm{Na}] 253.1236$, found 253.1230 . 
6m. ${ }^{1} \mathrm{H}$ NMR $\left(500 \mathrm{MHz}, \mathrm{CDCl}_{3}\right) \delta 4.77-4.70(\mathrm{~m}, 1 \mathrm{H}), 2.59-2.54(\mathrm{~m}, 1 \mathrm{H}), 2.43-2.31(\mathrm{~m}, 4 \mathrm{H})$, $2.17(\mathrm{~s}, 2 \mathrm{H}), 2.09(\mathrm{~s}, 3 \mathrm{H}), 1.73-1.62(\mathrm{~m}, 1 \mathrm{H}), 1.61(\mathrm{~s}, 3 \mathrm{H}), 1.59-1.47(\mathrm{~m}, 1 \mathrm{H}), 0.08(\mathrm{~s}, 1 \mathrm{H}) ;{ }^{13} \mathrm{C}$ NMR $\left(125 \mathrm{MHz}, \mathrm{CDCl}_{3}\right) \delta$ 208.66, 208.11, 146.07, 112.51, 49.05, 41.96, 41.47, 38.49, 30.00, $26.58,18.87,-1.03$.

6n. ${ }^{1} \mathrm{H}$ NMR $\left(500 \mathrm{MHz}, \mathrm{CDCl}_{3}\right) \delta 2.31$ (q, $\left.J=6.6 \mathrm{~Hz}, 1 \mathrm{H}\right), 2.09$ (s, 2H), 1.27-1.10 (m, 4H), $0.90(\mathrm{~d}, J=6.9 \mathrm{~Hz}, 3 \mathrm{H}), 0.77(\mathrm{t}, J=6.2 \mathrm{~Hz}, 3 \mathrm{H}), 0.01(\mathrm{~s}, 9 \mathrm{H}) ;{ }^{13} \mathrm{C} \mathrm{NMR}\left(125 \mathrm{MHz}, \mathrm{CDCl}_{3}\right) \delta$ 212.70, 49.11, 46.86, 35.17, 20.38, 15.91, 13.95, -1.16.

6o. $7.33-7.30(\mathrm{~m}, 2 \mathrm{H}), 7.24-7.20(\mathrm{~m}, 3 \mathrm{H}), 3.93(\mathrm{~s}, 2 \mathrm{H}), 3.51(\mathrm{t}, J=6.4 \mathrm{~Hz}, 2 \mathrm{H}), 2.76(\mathrm{t}, J=7.7$ $\mathrm{Hz}, 2 \mathrm{H}), 2.35$ (s, 2H), 2.01-1.96 (m, 2H), 0.19 (s, 9H); $\left.{ }^{13} \mathrm{C} \mathrm{NMR} \mathrm{(125} \mathrm{MHz,} \mathrm{CDCl}_{3}\right) \delta 207.89$, $141.76,128.52,128.47,125.96,76.61,70.71,34.16,32.39,31.32,-0.86$.

\section{Acknowledgements}

The authors thank UIC and the donors of American Chemical Society Petroleum Research Fund for financial support of this work. The mass spectrometry facility at UIUC and Mr. Furong Sun are greatly acknowledged.

\section{References}

1. Enders, D.; Lohray, B. B. Angew. Chem. 1988, 100, 594; Angew. Chem. Int. Ed. Engl. 1988, 27, 581. (b) Lohray, B. B.; Zimbinski, R. Tetrahedron Lett. 1990, 31, 7273. (c) Enders, D.; Prokopenko, O. F.; Raabe, G.; Runsink, J. Synthesis 1996, 1095. (d) Enders, D.; Otten, T. Synlett 1999, 747. (e) Enders, D.; Ward, D.; Adam, J.; Raabe, G. Angew. Chem. 1996, 108, 1059; Angew. Chem. Int. Ed. Engl. 1996, 35, 981. (f) Enders, D.; Fey, P.; Schmitz, T.; Lohray, B. B.; Jandeleit, B. J. Organomet. Chem. 1981, 5144, 227. (g) Enders, D.; Potthoff, M.; Raabe, G. Runsink, J. Angew. Chem. 1997, 109, 2454; Angew. Chem. Int. Ed. Engl. 1996, 36, 2362. (h) Enders, D.; Klein, D.; Raabe, G.; Runsink, J. Synlett 1997, 1271. (i) Hett, R. Diplomarbeit, RWTH Aachen 1988. (j) Enders, D.; Poiesz, C.; Joseph, R. Tetrahedron Assymetry. 1998, 9, 3709. (k) Enders, D.; Klein, D. Synlett 1999, 719. (1) Enders, D.; Adam, J.; Klein, D.; Otten, T. Synlett 2000, 10, 1371. (m) Landais, Y. Science of Synthesis 2002, 4, 757. (n) Chiara, J. L.; García, Á.; Sesmilo, E.; Vacas, T. Org. Lett. 2006, 8, 3935.

2. (a) Demuth, M. Helv. Chim. Acta. 1978, 61, 3136. (b) Seitz, D. E.; Zapata, A. Synthesis $1981,557$.

3. Hauser, C. R.; Hance, C. R. J. Am. Chem. Soc. 1952, 74, 5091.

4. Larson, G. L.; de Lopez-Cepero, I. M.; Tores, L.-E. Tetrahedron Lett. 1984, 25, 1673.

5. (a) Sato, S.; Okada, H.; Matsuda, I.; Izumi, Y. Tetrahedron Lett. 1984, 25, 769. (b) Beißwenger, H.; Hanack, M. Tetrahedron Lett. 1982, 23, 403. (c) Fristad, W. E.; Bailey, T. 
R.; Paquette, L. A. J. Org. Chem. 1980, 45, 3028. (d) Yamamoto, K.; Tomo, Y.; Suzuki, S. Tetrahedron Lett. 1980, 21, 2861.

6. (a) Corey, E. J.; Enders, D. Chem. Ber. 1978, 111, 1362. (b) Corey, E. J.; Enders, D.; Bock, M. G. Tetrahedron Lett. 1976, 17, 7. (c) Hudrlik, P. F.; Kulkarni, A. K. J. Am. Chem. Soc. 1981, 103, 6251.

7. (a) Kowalski, C. J.; O’Dowd, M. L.; Burke, M. C.; Fields, K. W. J. Am. Chem. Soc. 1980, 102, 5411. (b) Sampson, P.; Wiemer, D. J. Chem. Soc., Chem. Commun. 1985, 1746.

8. (a) Gutsche, C. D.; Redmore, D. In Carbocyclic Ring Expansion Reactions; Hart, H.; Karabatsos, G. J. Eds.; Academic Press: New York, 1968, 81-98. (b) Krow, G. R. Tetrahedron 1987, 43, 3. (c) Dabrowski, J. A.; Moebius, D. C.; Wommack, A. J.; Kornahrens, A. F.; Kingsbury, J. S. Org. Lett. 2010, 12, 3598. (d) Sletten, E. M.; Nakamura, H.; Jewett, J. C.; Bertozzi, C. R. J. Am. Chem. Soc. 2010, 132, 11799. (d) Moebius, D. C.; Kingsbury, J. S. J. Am. Chem. Soc. 2009, 131, 878. (e) Wommack, A. J.; Moebius, D. C.; Travis, A. L.; Kingsbury, J. S. Org. Lett. 2009, 11, 3202. (f) Hashimoto, N.; Aoyama, T.; Shioiri, T. Tetrahedron Lett. 1980, 21, 4619. (g) Maruoka, K.; Concepcion, A. B.; Yamamoto, H. Synthesis 1994, 1283. (h) Maruoka, K.; Concepcion, A. B.; Yamamoto, H. Synlett 1994, 521. Recent examples of diastereoselective process, see: (i) Hashimoto, T.; Miyamoto, H.; Naganawa, Maruoka, K. J. Am. Chem. Soc. 2009, 131, 11280. (j) Hashimoto, T.; Naganawa, Y.; Maruoka, K. J. Am. Chem. Soc. 2008, 130, 2434. (k) Hashimoto, T.; Naganawa, Y.; Maruoka, K. J. Am. Chem. Soc. 2009, 131, 6614.

9. Aoyama, T.; Shiori, T. Synthesis. 1984. 3. 228.

10. For the preparation of silyl enol ether, this one-step method should be much more convenient and cost-effective compared to Aggarwal's method. See Aggarwal, V. K.; Sheldon, C. G.; Macdonald, G. J.; Martin, W. P. J. Am. Chem. Soc. 2002, 124, 10300.

11. Levesque, P.; Fournier, P. A. J. Org. Chem. 2010, 75, DOI: 10.1021/jo1016713.

12. Li, J.; Sun, C.; Lee, D. J. Am. Chem. Soc. 2010, 132, 6640. 